\title{
Síntomas respiratorios asociados con la exposición a la contaminación del aire en cinco localidades de Bogotá, 2008-2011, estudio en una cohorte dinámica
}

\author{
Rodrigo Sarmiento', Luis Jorge Hernández², Edna Katalina Medina', \\ Natalia Rodríguez ${ }^{1}$, Jesús Reyes ${ }^{3}$ \\ 1 Línea de Aire, Ruido y Radiación Electromagnética, Observatorio de Salud Ambiental, Área de Vigilancia en \\ ${ }^{3}$ Línea de Aire, Ruido y Radiación Electromagnética, Vigilancia Sanitaria y Ambiental, Dirección de Salud Pública,
} Salud Pública, Secretaría Distrital de Salud de Bogotá, Bogotá, D.C., Colombia

2 Facultad de Medicina, Universidad de los Andes, Bogotá, D.C., Colombia Hospital del Sur, Bogotá, D.C., Colombia

Introducción. En Bogotá, la morbilidad por enfermedad respiratoria en menores de cinco años aumenta a medida que lo hace la contaminación del aire.

Objetivo. El objetivo de este estudio fue conocer la incidencia de los síntomas respiratorios en cinco localidades de Bogotá y su asociación con otros factores, entre 2008 y 2011.

Materiales y métodos. Se hizo un estudio de cohorte dinámica. El tamaño de la muestra fue de 3.278 niños de cinco localidades, divididos en dos grupos de acuerdo con la exposición a material en partículas. Se hizo el seguimiento de la aparición de diez síntomas respiratorios mediante un diario de registro. El análisis estadístico incluyó el cálculo de la razón de tasas de incidencia (RTI) y la regresión de Poisson para cada síntoma evaluado.

Resultados. La tos se presentó con mayor frecuencia en los niños más expuestos $\left(R T I=1,23 ; I_{95 \%}\right.$ $1,13-1,34)$ y la incidencia de expectoración fue mayor en ellos ( $\left.R T I=1,64 ; C_{95 \%} 1,47-1,84\right)$. En cuanto a las sibilancias, se observó una asociación con la exposición a material en partículas ( $R R=2,29$; $\left.I_{95 \%} 1,78-3,00\right)$, la presencia de fábricas en la vivienda $\left(R R=1,29 ; \quad I C_{95 \%} 1,01-1,68\right)$ y la edad $\left(\mathrm{RR}=0,95 ; \mathrm{IC}_{95 \%} 0,94-0,96\right)$. La proporción de riesgo atribuible a la exposición a la contaminación del aire, fluctuó según los síntomas entre 37 \% para las sibilancias nocturnas $\left(\mathrm{IC}_{95 \%} 26-47,8\right)$ y $10,6 \%$ para la tos $\left(\mathrm{IC}_{95 \%} 6,3-15\right)$.

Conclusión. Los efectos de la contaminación del aire extramuros se vieron potenciados por las condiciones socioeconómicas en las que vive y trabaja la población de Bogotá. Es necesario adoptar una aproximación conceptual más amplia desde la perspectiva de la salud pública y fortalecer la acción intersectorial para reducir los efectos de la contaminación del aire en la salud de la población.

Palabras clave: asma, enfermedades respiratorias, contaminación del aire, material en partículas, contaminación por humo de tabaco, aire en exteriores.

doi: http://dx.doi.org/10.7705/biomedica.v35i0.2445

Respiratory symptoms associated with air pollution in five localities of Bogotá, 2008-2011, a dynamic cohort study

Introduction: High levels of air pollution increase respiratory morbidity in children under five years of age.

Objective: To know the incidence of respiratory symptoms and its associated factors in five localities of Bogota.

Materials and methods: A dynamic cohort study was undertaken with a sample size of 3,278 children from five localities split into two groups according to the degree of exposure to particulate matter. Monitoring was conducted at the outbreak of ten respiratory symptoms through a diary of those symptoms. Statistical analysis included incidence rate ratio (IRR) calculations and Poisson regression models for each assessed symptom.

Results: Cough was more frequent in the highly exposed group (IRR=1.23, Cl 95\% 1.13-1.34). The most exposed group had higher incidence of expectoration ( $\mathrm{RR}=1.64, \mathrm{Cl}=95 \% 1.47-1.84)$. Wheezing

\section{Contribución de los autores:}

Rodrigo Sarmiento y Luis Jorge Hernández: diseño del estudio

Edna Katalina Medina y Natalia Rodríguez: coordinación del trabajo de campo y validación de la información

Jesús Reyes y Rodrigo Sarmiento: análisis estadístico

Todos los autores participaron por igual en la interpretación y discusión de los resultados, así como en la redacción del manuscrito. 
was associated with high exposure to particulate matter ( $R R=2.29, \mathrm{Cl} 95 \%$ 1.78-3.00), cottage industries ( $R R=1.29, \mathrm{Cl} 95 \%$ 1.01-1.68) and age ( $R R=0.95, \mathrm{Cl} 95 \%$ 0.94-0.96). Risk attributable to air pollution exposure fluctuated according to symptoms from 37\% for nocturnal wheezing (Cl 95\% 26-47.8) to 10.6 for cough (Cl 95\% 6.3-15\%).

Conclusion: Extradomiciliary air pollution effects are potentiated by socioeconomic living and working conditions of people in Bogota. A broader conceptual public health approach and the strengthening of intersector actions are required to reduce health effects of air pollution.

Key words: Asthma, respiratory tract diseases, air pollution, particulate matter, tobacco smoke pollution, outdoor air.

doi: http://dx.doi.org/10.7705/biomedica.v35i0.2445

La contaminación del aire es uno de los factores asociados a la enfermedad respiratoria, en particular, en menores de cinco años (1). De hecho, los niveles elevados de contaminantes en el aire, como el ozono troposférico, el material en partículas y el dióxido de nitrógeno, aumentan las hospitalizaciones por urgencias y las muertes debidas a la enfermedad respiratoria (2-4).

La contaminación del aire afecta particularmente a grupos etarios y poblaciones específicas. Por ejemplo, los menores de cinco años son especialmente vulnerables ante los efectos nocivos de la contaminación del aire, debido a sus características anatómicas y fisiológicas (5). Además, la enfermedad respiratoria es un factor ligado al ausentismo escolar en zonas con gran contaminación atmosférica (6). Las infecciones de las vías respiratorias inferiores son la principal causa de mortalidad en menores de cinco años en Colombia, y Bogotá es una de las ciudades más afectadas.

Además, la contaminación extramuros e intramuros está clasificada como uno de los factores de riesgo más importantes de mortalidad prematura y discapacidad en el país $(7,8)$.

Por otro lado, Bogotá es una de las ciudades menos equitativas del mundo (índice de Gini=0,61), solo superada por varias ciudades de Sudáfrica de acuerdo con datos del 2005. Para ese año, Colombia se ubicó en el grupo de países con "muy alta inequidad" (países cuyo coeficiente de Gini está en el rango de 0,50 a 0,59) (9), y desde 1990 , la brecha de la inequidad ha aumentado en un $24 \%$, presentando el peor porcentaje entre las ciudades latinoamericanas (9).

Correspondencia:

Rodrigo Sarmiento, Carrera 23 N ${ }^{\circ}$ 118-95, apartamento 502 , Bogotá, D.C., Colombia

Teléfono: (571) (314) 2538562

sarmientorodrigo@hotmail.com

Recibido: 02/07/14; aceptado: 14/05/15
Bogotá, la ciudad de mayor densidad de población de Latinoamérica, está dividida en 20 localidades con un alto nivel de segregación socio-espacial y una tendencia creciente de la informalidad laboral (alrededor del $45 \%$ de la población trabajadora está vinculada a trabajos informales) (10). Como resultado, el ambiente urbano se ha deteriorado seriamente durante la última década.

Según los datos reportados por las autoridades ambientales y los límites excedidos con respecto a la norma establecida en la Resolución 610 de 2010, el principal contaminante en Bogotá es el material en partículas, el cual tiene efectos directos sobre el aparato respiratorio e interactúa con los virus respiratorios, potenciando sus efectos (11). Según la Organización Mundial de la Salud, Bogotá es una de las ciudades de Latinoamérica más contaminadas con material en partículas de menos de $10 \mu \mathrm{m}\left(\mathrm{PM}_{10}\right)$ y $2,5 \mu \mathrm{m}\left(\mathrm{PM}_{2,5}\right)$, y los promedios anuales no cumplen con las recomendaciones para la protección de la población $\left(\mathrm{PM}_{10}=48 \mu \mathrm{g} /\right.$ $\mathrm{m}^{3}$, valor de referencia $20 \mu \mathrm{g} / \mathrm{m}^{3}$, y $\mathrm{PM}_{2,5}=27 \mu \mathrm{g} /$ $\mathrm{m}^{3}$, valor de referencia $10 \mu \mathrm{g} / \mathrm{m}^{3}$ ) (12). Además, los valores anuales de $\mathrm{PM}_{25}$ superan lo establecido por la norma nacional (valor de referencia $25 \mu \mathrm{g} /$ $\mathrm{m}^{3}$ ), mientras que los valores de $\mathrm{PM}_{10}$ se acercan al umbral fijado para este contaminante (valor de referencia $\left.50 \mu \mathrm{g} / \mathrm{m}^{3}\right)$.

Sumado a esto, debe mencionarse que, aunque la mayoría de las emisiones de material en partículas se debe al humo de la combustión de los automóviles expelida por los tubos de escape, la Red de Monitoreo de la Calidad del Aire de Bogotá no está midiendo el carbono negro, el cual tiene efectos graves en el sistema respiratorio $(13,14)$. Esto significa que el impacto negativo en la salud asociado al tráfico vehicular en Bogotá, no se conoce con certeza. Sin embargo, varios estudios llevados a cabo en el país han demostrado los efectos nocivos de la contaminación atmosférica. Según un estudio hecho en Bogotá en 1999, el 
aumento de $10 \mu \mathrm{g} / \mathrm{m}^{3}$ en los niveles de material en partículas se asoció con un incremento de las consultas de urgencia por enfermedad respiratoria (15). Además, la tendencia decreciente de la mortalidad por neumonía en menores de cinco años, se ha estancado durante el último año en 10 casos por cada 100.000 habitantes (16).

En Bogotá, las localidades más contaminadas, como Kennedy, Puente Aranda, Fontibón y Suba, son las que presentan un peor panorama en cuanto a morbilidad y mortalidad por enfermedad respiratoria (17). En el 2007, se hizo un estudio para evaluar 619 niños en jardines infantiles de las localidades de Kennedy, Puente Aranda y Fontibón, divididos en dos grupos en cada localidad según la exposición a $\mathrm{PM}_{10}$, y se encontró que la incidencia de síntomas respiratorios, como la tos, las sibilancias y la expectoración en los niños más expuestos, era mayor que en los no expuestos; asimismo, se observó que en varios de los jardines infantiles evaluados los niveles de contaminación intramuros eran, incluso mayores, que los valores exteriores (17).

Estos hallazgos coinciden con los de estudios previos, que han demostrado una exacerbación del asma bronquial y la enfermedad respiratoria asociada al aumento en los niveles de contaminación del aire (18). Sin embargo, se sabe poco sobre la incidencia de los síntomas respiratorios, la enfermedad respiratoria y el asma bronquial originados por otras fuentes de contaminación, como la humedad y el moho en la vivienda, el humo de segunda mano o el uso de combustibles sólidos para cocinar, y sus potenciales interacciones con la contaminación extramuros y los efectos en la frecuencia y la gravedad de la enfermedad respiratoria.

El objetivo de este estudio fue conocer la incidencia de los síntomas respiratorios en cinco localidades de Bogotá y su asociación con distintas fuentes de contaminación intramuros y extramuros en el periodo comprendido entre 2008 y 2011.

\section{Materiales y métodos}

\section{Población de estudio}

En un estudio de cohorte llevado a cabo entre 2008 y 2011, se evaluaron niños menores de cinco años en cinco localidades de Bogotá (Puente Aranda, Kennedy, Fontibón, Suba y Tunjuelito) identificadas por la autoridad ambiental como áreas generadoras de contaminación con material en partículas.
El tamaño de la muestra fue de 3.278 niños (1.718 en la cohorte de mayor exposición y 1.580 en la cohorte de menor exposición), para un nivel de confianza del $95 \%$, una potencia de $80 \%$ y una incidencia esperada de morbilidad respiratoria del $30 \%$, cálculos hechos con el programa EpiInfo 6.4 .

La muestra se seleccionó entre niños pertenecientes a 17 jardines infantiles públicos en dichas localidades; los menores eran elegibles si pasaban la mayor parte de su tiempo en el jardín (7 a.m. a 5 p.m.). La muestra se ajustó en un $15 \%$ para dar cuenta de posibles pérdidas. La cohorte fue dinámica, y varió según el año y la localidad.

\section{Zonas de exposición}

Se obtuvo el censo de los jardines infantiles públicos de las cinco localidades. Se seleccionó una muestra de jardines con mayor exposición y otra con menor exposición a la contaminación del aire por material en partículas. Los criterios utilizados para la clasificación en el grupo de alta exposición, fueron la ubicación del jardín a una distancia menor de $100 \mathrm{~m}$ de las principales fuentes fijas 0 móviles emisoras de este contaminante como: (i) vías en mal estado, (ii) vías de alto flujo vehicular, (iii) presencia de chimeneas y (iv) polígonos industriales. Los jardines que no presentaban ninguno de estos criterios se clasificaron como de menor exposición.

\section{Instrumentos}

Después de tener la autorización mediante consentimiento informado, se aplicó una encuesta inicial de caracterización de los niños con el instrumento desarrollado inicialmente en el estudio Asthma and Persistent Wheeze in the Harvard Six Cities, con ajustes incorporados a partir de la encuesta ISAAC (International Study on Asthma and Allergies in Childhood) y su adaptación en Colombia $(19,20)$. Dicho instrumento recopila información sobre la morbilidad sentida y los aspectos socioeconómicos, así como los factores asociados intramuros y extramuros, es decir, las condiciones existentes tanto en el jardín infantil como en las viviendas de los menores.

Para el seguimiento de la cohorte se utilizó el diario de síntomas validado previamente para Colombia (17). Por medio de este instrumento, se hizo el seguimiento de los síntomas respiratorios en niños menores de cinco años, midiendo la presencia de síntomas respiratorios de manera diaria en el jardín u hogar infantil seleccionado, de 
lunes a viernes, mediante la observación directa del encuestador, y los fines de semana, mediante entrevista telefónica.

Se evaluaron los siguientes eventos: tos, expectoración o flema, sibilancias, disnea, obstrucción nasal, secreción nasal, enrojecimiento o rasquiña en los ojos, y ausentismo escolar por enfermedad respiratoria. Se entrenó a los encuestadores, se diseñó un folleto instructivo y se estandarizaron las definiciones de caso para reducir el sesgo del entrevistador.

\section{Análisis estadístico}

Para el análisis estadístico se obtuvieron las frecuencias absolutas y relativas de cada una de las variables de la encuesta de caracterización. Para el análisis bivariado se utilizó la prueba de ji al cuadrado. En cuanto a la cohorte, se calcularon las tasas de incidencia y las razones de las tasas de incidencia con intervalos de confianza del $95 \%\left(\mathrm{IC}_{95 \%}\right)$ con el programa Open-Epi. Por último, se hizo un análisis multivariado con modelos de regresión de Poisson mediante el programa SPSS $20^{\mathrm{TM}}$, para evaluar la asociación entre las variables analizadas y las tasas de incidencia de los síntomas, empleando la razón de tasas o riesgo relativo (RR). Estos modelos se construyeron a partir de las asociaciones significativas para cada uno de los síntomas, obtenidas en las fases del análisis descriptivo y bivariado.

\section{Consideraciones éticas}

Los padres o los cuidadores de los participantes dieron su autorización para la participación de sus hijos o de los menores bajo su responsabilidad, mediante consentimiento informado verbal y escrito. Este estudio recibió la aprobación del Comité de Ética de la Universidad Nacional de Colombia.

\section{Resultados}

\section{Análisis descriptivo}

La distribución por sexo arrojó un resultado de $56 \%$ de niños ( $n=1.825)$ y $44 \%$ de niñas $(n=1.453)$. La edad promedio fue de 33 meses (desviación estándar=12 meses). La distribución de la muestra por año se presenta en el cuadro 1.

Las características sociodemográficas y otros factores de exposición intramuros y extramuros, fueron similares en ambos grupos. Cabe resaltar la gran prevalencia de sibilancias en estas localidades (grupo de mayor exposición $=41 \%$, grupo de menor exposición=38 \%). Además, se observó una proporción elevada de contaminantes en intramuros, en particular por exposición al humo de tabaco (grupo de mayor exposición: $29 \%$, grupo de menor exposición: $32 \%)$, y a la humedad o el moho en la vivienda (grupo de mayor exposición: $38 \%$, grupo de menor exposición: $39 \%$ ). Además, se encontró una prevalencia elevada de exposición al tráfico pesado y a vías en mal estado. Con respecto a otras variables, la proporción de bajo peso al nacer fue de $14 \%$ (grupo de mayor exposición: $13 \%$, grupo de menor exposición: $15 \%$ ), y la de cama compartida o lecho compartido fue de $50 \%$ (grupo de mayor exposición: $49 \%$, grupo de menor exposición: $50 \%$ ). Estas características se presentan en el cuadro 2.

Con relación a la incidencia de los síntomas respiratorios, se observó que en el grupo de mayor exposición la tos se presentó con mayor frecuencia, con una razón de tasas de incidencia (RTI) de 1,23 (IC ${ }_{95 \%}$ 1,13-1,34); igualmente, en el grupo más expuesto hubo mayor incidencia de expectoración ( $\left.R T I=1,64 ; \quad I C_{95 \%} 1,47-1,84\right)$, así como de sibilancias diurnas $\left(\mathrm{RTl}=1,79 ; \mathrm{IC}_{95 \%} 1,40\right.$ $2,28)$ y nocturnas $\left(R T I=2,19 ; I_{95 \%} 1,70-2,83\right)$. En los niños asistentes a jardines expuestos a altos niveles de contaminación del aire, se presentó más ausentismo escolar por enfermedad respiratoria $\left(R T I=1,32 ; \quad I C_{95 \%}\right.$ 1,04-1,67). Por otro lado, los síntomas de las vías respiratorias superiores, como la obstrucción nasal ( $R T I=0,89 ; \mathrm{IC}_{95 \%} 0,80$ 0,98 ) y el enrojecimiento de los ojos, tuvieron una mayor incidencia en el grupo de menor exposición ( $\left.R T I=0,75 ; I_{95 \%} 0,56-0,99\right)$. Los hallazgos mencionados se presentan en el cuadro 3.

\section{Análisis multivariado}

De acuerdo con la regresión de Poisson, las zonas identificadas como de alto nivel de exposición al material en partículas, se asociaron con mayor

Cuadro 1. Síntomas respiratorios por cohorte y año debidos a la contaminación del aire en cinco localidades de Bogotá

\begin{tabular}{cccr}
\hline Año & $\begin{array}{c}\text { Grupo de mayor } \\
\text { exposición }\end{array}$ & $\begin{array}{c}\text { Grupo de menor } \\
\text { exposición }\end{array}$ & Total \\
\hline 2008 & 393 & 404 & 797 \\
2009 & 449 & 431 & 880 \\
2010 & 539 & 492 & 1.031 \\
2011 & 337 & 233 & 570 \\
Total & 1.718 & 1.560 & 3.278 \\
\hline
\end{tabular}

Fuente: Sistema de información sobre aire, ruido y radiación electromagnética, Secretaría de Salud de Bogotá

Jardines infantiles de mayor exposición: 8 (2 en Kennedy, 2 en Puente Aranda, 2 en Fontibón, 1 en Suba, 1 en Tunjuelito)

Jardines infantiles de menor exposición: 9 (2 en Kennedy, 2 en Puente Aranda, 2 en Fontibón, 2 en Suba, 1 en Tunjuelito) 
Cuadro 2. Caracterización sociodemográfica por cohorte y síntomas respiratorios en cinco localidades de Bogotá

\begin{tabular}{|c|c|c|}
\hline Variable & $\begin{array}{l}\text { Grupo de mayor exposición } \\
\qquad n=1.718(50,8 \%)\end{array}$ & $\begin{array}{c}\text { Grupo de menor exposición } \\
n=1.560(49,2 \%)\end{array}$ \\
\hline \multicolumn{3}{|l|}{ Sexo } \\
\hline Masculino & $967(54,7)$ & $952(45,7)$ \\
\hline Femenino & $800(45,3)$ & $758(44,3)$ \\
\hline \multicolumn{3}{|l|}{ Edad (años) } \\
\hline Media & $33,7(\mathrm{DE}=2,3)$ & $33,2(\mathrm{DE}=11,7)$ \\
\hline Prevalencia de síntomas respiratorios & n \% & n \% \\
\hline Prevalencia de sibilancias alguna vez & $704(41)$ & $610(38)$ \\
\hline Prevalencia de sibilancias en el último año & $692(41)$ & $620(39)$ \\
\hline Prevalencia de tos en la noche & $616(36)$ & $552(35)$ \\
\hline Proporción de ausentismo escolar & $555(33)$ & $551(35)$ \\
\hline Algún contacto del niño con fumadores & $497(29)$ & $502(32)$ \\
\hline Madre fumó durante el embarazo & $174(10)$ & $136(9)$ \\
\hline Madre fumó durante los dos primeros años de vida del niño & $274(16)$ & $174(11)$ \\
\hline Fábrica en la vivienda & $317(19)$ & $2012(13)$ \\
\hline Humedad en la vivienda & $642(38)$ & $607(39)$ \\
\hline Presencia de ratones en la vivienda & $243(14)$ & $166(11)$ \\
\hline Presencia de cucarachas en la vivienda & $108(6)$ & 120 (8) \\
\hline Chimeneas a menos de $100 \mathrm{~m}$ & $601(35)$ & $616(39)$ \\
\hline Presencia de tráfico pesado & $998(58)$ & $9092(57)$ \\
\hline Edificaciones en construcción/deterioro de la malla vial & 797 (47) & $715(46)$ \\
\hline Bajo peso al nacer & $228(13)$ & $238(15)$ \\
\hline Lecho compartido & $831(49)$ & $792(50)$ \\
\hline
\end{tabular}

Fuente: Sistema de información sobre aire, ruido y radiación electromagnética, Secretaría de Salud de Bogotá

Cuadro 3. Incidencia de los síntomas respiratorios en niños de menos de cinco años de edad en cinco localidades de Bogotá, 2008-2011

\begin{tabular}{|c|c|c|c|c|c|c|c|}
\hline \multirow[t]{2}{*}{ Síntomas } & \multicolumn{2}{|c|}{ Grupo de mayor exposición } & \multirow{2}{*}{$\begin{array}{c}\text { Tasa de } \\
\text { incidencia } \\
\left({ }^{*} 10.000\right)\end{array}$} & \multicolumn{2}{|c|}{ Grupo de menor exposición } & \multirow{2}{*}{$\begin{array}{c}\text { Tasa de } \\
\text { incidencia } \\
\left({ }^{*} 10.000\right)\end{array}$} & \multirow{2}{*}{$\begin{array}{c}\text { Razón de tasas } \\
\text { (RTI) } \\
\left(\mathrm{IC}_{95 \%}\right)\end{array}$} \\
\hline & Eventos & $\begin{array}{c}\text { Días de } \\
\text { exposición }\end{array}$ & & Eventos & $\begin{array}{c}\text { Días de } \\
\text { exposición }\end{array}$ & & \\
\hline Secreción nasal & 3.461 & 144.668 & 239,2 & 3.470 & 151.735 & 228,7 & $1,04(0,99-1,09)$ \\
\hline Tos & 1.202 & 214.501 & 56,04 & 915 & 201.170 & 45,4 & $1,23(1,13,-1,34$ \\
\hline Expectoración & 841 & 239.357 & 35,14 & 483 & 226.132 & 21,36 & $1,64(1,47-1,84)$ \\
\hline Obstrucción nasal & 739 & 238.376 & 31,0 & 775 & 222.751 & 34,79 & $0,89(0,80-0,98)$ \\
\hline Ausentismo escolar & 168 & 244.179 & 6,88 & 119 & 229.086 & 5,19 & $1,32(1,04-1,67)$ \\
\hline Sibilancias diurnas & 186 & 241.029 & 7,7 & 98 & 227.315 & 4,3 & $1,79(1,40-2,28)$ \\
\hline Sibilancias nocturnas & 190 & 202.007 & 9,40 & 86 & 200.723 & 4,28 & $2,19(1,70-2,83)$ \\
\hline $\begin{array}{l}\text { Enrojecimiento de } \\
\text { ojos/sensación } \\
\text { pruriginosa ocular }\end{array}$ & 90 & 244.298 & 3,68 & 112 & 228.403 & 4,90 & $0,75(0,56-0,99)$ \\
\hline Disnea & 48 & 207.607 & 2,31 & 65 & 198.488 & 3,27 & $0,70(0,48-1,02)$ \\
\hline
\end{tabular}

Fuente: Sistema de información sobre aire, ruido y radiación electromagnética, Secretaría de Salud de Bogotá

frecuencia de la mayoría de los síntomas evaluados, con excepción del enrojecimiento de los ojos. También, se observó que cuanto menor era la edad, la frecuencia de síntomas respiratorios aumentaba.

La tos se asoció con las siguientes variables: las zonas de alta exposición a material en partículas $\left(R R=1,15 ; I C_{95 \%} 1,06-1.24\right)$; la edad $\left(R R=0,98 ; I_{95 \%}\right.$ $0,98-0,99)$, y la exposición al humo de segunda mano $\left(R R=1,35 ; I_{95 \%} 1,23-1,49\right)$.
Con relación a las sibilancias, se observó una asociación con la exposición a material en partículas $\left(R R=2,29 ; I_{95 \%} 1,78-3,00\right)$, la presencia de fábricas en el interior de la vivienda $(R R=1,29$; $\left.I_{95 \%} 1,01-1,68\right)$ y la edad $\left(R R=0,95 ; \quad I C_{95 \%}\right.$ $0,94-0,96)$. Además, $32 \%$ del grupo de mayor exposición presentó ausentismo por enfermedad respiratoria $\left(R R=1,32 ; I_{95 \%} 1,02-1,72\right)$.

Cabe resaltar que no se encontró asociación entre el síntoma de enrojecimiento de los ojos y los niños 
que acudían al jardín infantil más expuesto, aunque sí entre este síntoma y la exposición al humo de segunda mano ( $\left.R R=2,04 ; I_{95 \%} 1,34-3,25\right)$. En el cuadro 4 se presentan los modelos que más se ajustaron a cada uno de los síntomas evaluados.

Con relación a la proporción de riesgo atribuible a la exposición a la contaminación del aire en la población, se encontró que dicha proporción fluctuó de acuerdo con los síntomas entre $37 \%$ para las sibilancias nocturnas $\left(\mathrm{IC}_{95 \%} 26-47,8 \%\right)$ y $10,6 \%$ para la tos $\left(\mathrm{IC}_{95 \%}, 6,3-15 \%\right)$. Por lo tanto, las sibilancias fueron el síntoma respiratorio más asociado a la exposición a altos niveles de contaminación del aire por material en partículas. Los resultados de los cálculos de la proporción de los riesgos atribuibles se presentan en el cuadro 5.

\section{Discusión}

Los síntomas respiratorios asociados a las vías respiratorias inferiores, como la tos y las sibilancias, tuvieron una mayor relación con la exposición a niveles elevados de material en partículas en el jardín infantil. Se presentó 15 \% más de casos de tos y más del doble de casos de sibilancias $\left(R R=2,29 ; \quad I C_{95 \%}, 1,78-3,00\right)$ en el grupo más expuesto en comparación con el menos expuesto. Este efecto se vio potenciado por la exposición a factores de contaminación intramuros, como el humo de segunda mano y la presencia de fábricas en el interior de las viviendas. Algunos factores asociados a las características socioeconómicas y a ciertos hábitos (lecho compartido y el antecedente de bajo peso al nacer), también tuvieron relación con una mayor incidencia de síntomas respiratorios. Además, si bien varias fuentes de exposición a la contaminación extramuros situadas a menos de $100 \mathrm{~m}$ de la vivienda, como la circulación de tráfico pesado (buses, busetas y camiones), no tuvieron un impacto sobre la incidencia de los síntomas respiratorios de manera aislada, el efecto agregado de la exposición a material en partículas en el jardín infantil se asoció con un incremento de los síntomas. Estos hallazgos concuerdan con lo que se conoce sobre los efectos de la contaminación por material en partículas y los síntomas respiratorios.

Cuadro 4. Modelos de regresión de Poisson aplicados a los síntomas respiratorios en cinco localidades de Bogotá, 2008-2011

\begin{tabular}{llccc}
\hline & \multicolumn{1}{c}{ Factores asociados } & Coeficiente $\boldsymbol{\beta}$ & Riesgo relativo (RR) & IC $_{95 \%}$ \\
\hline Tos & Exposición a material en partículas & 0,14 & 1,15 & $(1,06-1,24)$ \\
& Edad & $-0,017$ & 0,98 & $(0,98-0,99)$ \\
Sibilancias & Exposición a humo de segunda mano & 0,30 & 1,35 & $(1,23-1,49)$ \\
& Exposición a material en partículas & 0,83 & 2,29 & $(1,78-3,00)$ \\
& Fábrica en el interior de la vivienda & 0,26 & 1,29 & $(1,01-1,68)$ \\
& Bajo peso al nacer & 0,28 & 1,32 & $(0,97-1,77)$ \\
Expectoración & Edad & $-0,05$ & 0,95 & $(0,94-0,96)$ \\
& Exposición a material en partículas & 0,59 & 1,80 & $(1,59-2,05)$ \\
& Edad & $-0,02$ & 0,97 & $(0,96-0,98)$ \\
& Lecho compartido & 0,38 & 1,47 & $(1,29-1,68)$ \\
Enrojecimiento de ojos/ & Tráfico pesado a menos de 100 m & 0,22 & 1,25 & $(1,09-1,44)$ \\
sensación pruriginosa ocular & Exposición a humo de segunda mano & 0,71 & & \\
Ausentismo escolar por & Edad & $-0,017$ & 2,04 & $(1,34-3,25)$ \\
enfermedad respiratoria & Exposición a material en partículas & 0,28 & 0,98 & $(0,97-0,99)$ \\
& Edad & $-0,01$ & 1,32 & $(1,02-1,72)$ \\
& & 0,98 & $(0,97-0,99)$ \\
\hline
\end{tabular}

Fuente: Sistema de información sobre aire, ruido y radiación electromagnética, Secretaría de Salud de Bogotá

Cuadro 5. Porcentaje de riesgo atribuible a síntomas respiratorios por exposición a la contaminación con material en partículas en cinco localidades de Bogotá, D.C., 2008-2011

\begin{tabular}{|c|c|c|c|c|}
\hline Síntomas & $\begin{array}{l}\text { Tasa de incidencia } \\
\text { global }\left({ }^{*} 10.000\right)\end{array}$ & $\begin{array}{l}\text { Diferencia de } \\
\text { riesgo }\left({ }^{*} 10.000\right)\end{array}$ & $\begin{array}{l}\text { Fracción atribuible en } \\
\text { expuestos }\left(\mathrm{IC}_{95 \%}\right)\end{array}$ & $\begin{array}{c}\text { Porcentaje de riesgo } \\
\text { atribuible a la población ( }\left(\mathrm{IC}_{95 \%}\right)\end{array}$ \\
\hline Tos & 50,93 & 10,5 & $18,8(11,5-25,5)$ & $10,6 \quad(6,3-15)$ \\
\hline Sibilancias diurnas & 6,06 & 3,40 & $44 \quad(28,6-56,2)$ & $28,9(17,1-39,8)$ \\
\hline Sibilancia nocturnas & 6,85 & 5,12 & $54,4(41,2-64,6)$ & $37,4 \quad(26-47,8)$ \\
\hline Expectoración & 28,44 & 13,78 & $39,2 \quad(32-45,6)$ & $24,9(19,4-30,1)$ \\
\hline Ausentismo escolar & 6,06 & 1,68 & $24,5 \quad(4,5-40,3)$ & $14,3 \quad(2,3-25,8)$ \\
\hline
\end{tabular}

Fuente: Sistema de información sobre aire, ruido y radiación electromagnética, Secretaría de Salud de Bogotá 
En un estudio llevado a cabo en Ámsterdam, se encontró que los niños que vivían cerca de carreteras o vías muy transitadas tenían $35 \%$ más de exposición personal al hollín que los que habitaban en un lugar apartado, lo cual se pudo observar en el presente estudio al constatar que los niños expuestos al tráfico pesado a escasa distancia de su vivienda tenían más riesgo de presentar expectoración (21). En Canadá, se encontró que los contaminantes relacionados con el tráfico aumentaban la probabilidad de padecer asma en niños menores de cuatro años (22).

Por otro lado, en un estudio de cohorte con seguimiento de 150 niños asmáticos de dos a seis años de edad, se demostró que había asociación entre el incremento de los niveles de material en partículas intramuros y la gravedad de los síntomas indicativos de asma en los niños que permanecían aproximadamente el $80 \%$ de su tiempo dentro de las instituciones educativas. Asimismo, se observó una mayor contaminación intramuros que extramuros, lo cual se relacionó con la cercanía a fuentes de emisión de material en partículas como las vías de alto flujo vehicular, hallazgo similar al del presente estudio (23).

Aunque este estudio se proponía evaluar la contaminación extramuros con material en partículas, en varios estudios se ha afirmado que la contaminación intramuros puede ser incluso mayor que la extramuros (23). Dicho efecto se observó también en otro estudio llevado a cabo en un jardín infantil de la localidad de Puente Aranda en Bogotá, en el cual, aunque los niveles encontrados no fueron mayores en el interior que en el exterior, sí fueron similares (intramuros: $86 \mu \mathrm{g} / \mathrm{m}^{3}$; extramuros: $\left.91 \mu \mathrm{g} / \mathrm{m}^{3}\right)(17)$.

Si bien el objetivo del presente estudio no fue determinar las causas de la contaminación intramuros, se han señalado como principales responsables el tabaquismo y la cercanía a fuentes de emisión fijas y móviles, lo que facilita la entrada de material en partículas en el jardín infantil e impide su dispersión. También, existe la posibilidad de que haya problemas de ventilación en los jardines infantiles, pero en el país no se han hecho estudios de este tipo.

Por otro lado, la exposición al humo de segunda mano representa un aspecto importante en la carga de morbilidad por síntomas respiratorios. En el presente estudio el tabaquismo pasivo demostró estar asociado con la presencia de tos y enrojecimiento de los ojos en los niños menores de cinco años. En estudios previos se han evidenciado los efectos negativos de esta exposición, ya que los niños expuestos al humo de segunda mano son más proclives a presentar bronquitis sibilantes en los primeros seis meses de vida (24). Asimismo, en otra investigación se encontró que los niños nacidos de madres que habían fumado durante el embarazo, eran más propensos a ser hospitalizados por bronquiolitis (25). Se estima que el $28 \%$ del total de muertes y el $61 \%$ de los años de vida ajustados por discapacidad, se pueden atribuir a la exposición al humo de segunda mano en niños, lo que obliga a fortalecer las medidas de implementación del Convenio Marco de la OMS para el Control del Tabaco a nivel mundial, con el fin de proteger a la población infantil (26).

En este estudio no se midieron los niveles de contaminantes biológicos presentes intramuros, como los ácaros y el moho, y la información sobre ellos se recogió únicamente mediante la encuesta. En el caso de Bucaramanga, la información disponible ha señalado una prevalencia elevada de hongos en los hogares, en particular de las especies Cladosporium (98\%) y Fusarium (82\%), así como de ácaros (29\%), predominantemente Dermatophagoides (16\%) (27).

En otro estudio en que se evaluó la contaminación intramuros en Colombia, se demostró una prevalencia de contacto con fumadores de tan solo $1,6 \%$ en Bucaramanga, en comparación con el $30 \%$ encontrado en este estudio. Al indagar sobre la humedad o el moho en la vivienda, la proporción de expuestos también fue mayor en Bogotá (39\%) que en Bucaramanga (26\%) (28). En consecuencia, las políticas públicas en Bogotá deberían diseñar intervenciones para la reducción de estas exposiciones. Por último, los síntomas respiratorios debidos a la contaminación extramuros se asociaron con el tráfico vehicular, siendo esta la principal fuente de emisión de material en partículas en ambas ciudades.

Según los datos obtenidos en este estudio, el $10 \%$ de los casos de tos en menores de cinco años podrían atribuirse a la exposición a altos niveles de material en partículas. Asimismo, el $28 \%$ de los casos de sibilancias en menores de cinco años podría explicarse por la exposición a material en partículas. Sin embargo, en Bogotá la contaminación del aire por material en partículas no es el único factor que contribuye a la aparición de síntomas respiratorios y al desarrollo de las enfermedades respiratorias, pues otros factores, como el lecho compartido, el tabaquismo pasivo, 
las fábricas del sector informal que funcionan dentro de la vivienda, la cercanía a edificaciones en construcción y el hacinamiento, se relacionaron claramente con dichos síntomas y fueron factores determinantes en un buen número de casos de ausentismo escolar.

En este estudio no se exploró el antecedente de la lactancia materna como factor protector contra los síntomas respiratorios y, aunque actualmente no parece haber un consenso en torno a este aspecto, algunas investigaciones hechas en Bucaramanga han demostrado una reducción en la incidencia de asma en menores que fueron lactados durante un periodo superior a tres meses (29). Además, los altos niveles de informalidad y el desempleo condicionan la proliferación de pequeñas industrias y fábricas en el interior de las viviendas, con los consecuentes efectos negativos sobre la salud producidos por la emisión de contaminantes.

Estas circunstancias socioeconómicas amplían la brecha de la inequidad y agravan la segregación socio-espacial de Bogotá, observándose una relación directa entre la injusticia social y la ambiental. El índice de desarrollo humano urbano es un indicador que permite evaluar la inequidad y que se obtiene con base en cuatro componentes: (i) los ingresos, (ii) la supervivencia de los niños menores de un año, (iii) la cobertura educativa, y (iv) la variable urbana que se compone del tiempo de movilidad y la disponibilidad de equipamientos. En ciertas localidades de alta contaminación, como Kennedy $(0,77)$, Suba $(0,80)$ y Tunjuelito $(0,76)$, este índice está por debajo del promedio de Bogotá $(0,81)(30)$. Por ello, las intervenciones en salud pública para contrarrestar los efectos de la contaminación del aire deberían abordarse desde un modelo conceptual que tenga en cuenta, no solo la relación entre la exposición y el efecto, sino también los factores estructurales determinantes intermedios y próximos.

La Organización Panamericana de la Salud ha propuesto la metodología de "fuerzas motrices", la cual identifica varios niveles; el nivel superior, donde se sitúan las "fuerzas motrices" y las "presiones" generadas sobre el medio ambiente, lo que a su vez determina el "estado", representado en el entorno en el cual ocurre la "exposición" de las personas, que son quienes, a la postre, sufren los "efectos" en la salud.

Como ya se mencionó, en el caso de los síntomas respiratorios asociados a la contaminación del aire en Bogotá, se observó que la exposición estaba determinada por factores socioeconómicos y ambientales (bajo peso al nacer, humo de segunda mano, fábricas en las viviendas, combustibles no limpios), los cuales están determinados, a su vez, por factores estructurales como la inequidad, el desempleo o la pobreza. Esta metodología permite, además, determinar acciones dirigidas a modificar positivamente las fuerzas motrices, las presiones, el estado del entorno, así como a controlar las exposiciones de las poblaciones y a atender y superar sus efectos en la salud (31).

En el caso de Bogotá, la aplicación de dicha metodología a los resultados de este estudio, permite formular las siguientes recomendaciones de política pública para la reducción del impacto negativo de la contaminación del aire en la población infantil de Bogotá: (i) fortalecimiento de la vigilancia ambiental y los sistemas de alerta temprana en salud; (ii) educación sanitaria de padres y cuidadores; (iii) reubicación de jardines infantiles localizados en zonas de alta exposición a material en partículas; (iv) mejoramiento de vías; (v) reducción de la inequidad y de la segregación socio-espacial; (vi) uso de combustibles limpios, y (vii) estrategias para la reducción del consumo de tabaco en los hogares.

La principal limitación del estudio consistió en que la exposición del jardín infantil se clasificó solo con base en criterios de contaminación extramuros, lo que pudo haber resultado en una clasificación errónea de la población con mayor riesgo de presentar síntomas respiratorios. Se deberían tener en cuenta, no solo las variables externas a los jardines, sino también las exposiciones y las vulnerabilidades diferenciadas en su interior.

Otra de las limitaciones del estudio fue la subjetividad de los criterios utilizados para evaluar la exposición, por lo que en estudios posteriores se deberían incorporar metodologías de evaluación acumulada del riesgo o de vigilancia continua de los niveles de material en partículas $\left(\mathrm{PM}_{10} \mathrm{y}\right.$ $\mathrm{PM}_{2,5}$ ) intramuros y extramuros durante todo el periodo de seguimiento (32). De igual manera, la vigilancia de la exposición personal podría brindar una información más exacta sobre la relación dosis-respuesta.

Otra limitación de este estudio fue no haber hecho mediciones intramuros de la exposición al tabaco, la humedad en la vivienda o la presencia de ácaros, las cuales deberán evaluarse en futuras investigaciones. 
Se pudo observar que la incidencia de síntomas respiratorios graves como la tos y las sibilancias, fue mayor en menores de cinco años que asistían a jardines con altos niveles de contaminación del aire extramuros. No obstante, los efectos de la contaminación del aire se vieron potenciados por las condiciones sociales y económicas en las que vive y trabaja la población de Bogotá, caracterizadas por la inequidad social y ambiental. Por lo tanto, se requiere una aproximación conceptual más amplia desde la perspectiva de la salud pública y un fortalecimiento de la acción intersectorial, para reducir los efectos de la contaminación del aire en la salud de los menores de cinco años.

\section{Conflicto de intereses}

Los autores declaran no tener conflicto de intereses.

\section{Financiación}

El presente estudio fue financiado por la Secretaría Distrital de Salud de Bogotá.

\section{Referencias}

1. Gauderman WJ. Air pollution and children - an unhealthy mix. N Eng J Med. 2006;355:78-9. http://dx.doi.org/10.1056/ NEJMe068096

2. Gouveia N, Freitas CU, Martins LC, Marcilio IO. Respiratory and cardiovascular hospitalizations associated with air pollution in the city of São Paulo, Brazil. Cad Saúde Pública. 2006;22:2669-77. http://dx.doi.org/10.1590/S0102311X2006001200016

3. Dales RE, Cakmak S, Doiron MS. Gaseous pollutants and hospitalization for respiratory disease in the neonatal period. Environ Health Perspect. 2006;114:1751-4. http:// dx.doi.org/10.1289/ehp.9044

4. Pope CA, Dockery DW, Spengler JD, Raizenne ME. Respiratory health and PM 10 pollution: A daily time series analysis. Am Rev Resp Dis. 1991;144:668-74. http://dx.doi. org/10.1164/ajrccm/144.3_Pt_1.668

5. Ward D, Ayres I. Particulate air pollution and panel studies in children: A systematic review. Occup Environ Med. 2004;61:e13. http://dx.doi.org/10.1136/oem.2003.007088

6. Gilliland FD, Berhane K, Rappaport EB, Thomas D, Avol E, Gauderman WJ, et al. The effects of ambient air pollution on school absenteeism due to respiratory illness. Epidemiology. 2001;12:43-54.

7. Institute of Health Metrics and Evaluation. Global Burden of Disease Study 2012. Fecha consulta: 10 de abril de 2014. Disponible en: http://www.healthmetricsandevaluation.org/ gbd/visualizations/gbd-heatmap.

8. Vos T, Flaxman AD, Naghavi M, Lozano R, Michaud C, Ezzati M, et al. Years lived with disability (YLD) for 1160 sequelae of 289 diseases and injuries 1990-2010: A systematic analysis for the Global Burden of Disease Study 2010. Lancet. 2012;380:2163-96. http://dx.doi.org/10.1016/ S0140-6736(12)61729-2
9. United Nations Habitat. State of the world's cities 20102011: Bridging the urban divide 2012. Fecha de consulta: 15 de marzo de 2013. Disponible en: http://www.unhabitat.org/ pmss/listltemDetails.aspx?publicationID=2917.

10. Departamento Administrativo Nacional de Estadística. Resumen ejecutivo medición del empleo informal. Fecha de consulta: 13 de abril de 2014. Disponible en: https:// www.dane.gov.co/daneweb_V09/files/investigaciones/ boletines/ech/ech_informalidad/re_ech_informalidad_dic_ feb2012.pdf.

11. Lambert AL, Mangum JB, DeLorme MP, Everitt Jl. Ultrafine carbon black particles enhance respiratory syncytial virusinduced airway reactivity, pulmonary inflammation, and chemokine expression. Toxicol Sci. 2003;72:339-46. http:// dx.doi.org/10.1093/toxsci/kfg032

12. World Health Organization. Outdoor pollution database in cities 2014. Fecha de consulta: 15 de mayo de 2014. Disponible en: http://www.who.int/phe/health_topics/ outdoorair/databases/cities/en/.

13. Secretaría Distrital de Ambiente. Atlas ambiental de Bogotá, 2007. Fecha de consulta: 12 de abril de 2014. Disponible en: http://oab.ambientebogota.gov.co/resultado_ busquedas.php?AA_SL_Session=8cf97c692b\&x=3947.

14. Smith KR, Jerrett M, Anderson HR, Burnett RT, Stone $\mathrm{V}$, Derwent R, et al. Public health benefits of strategies to reduce greenhouse-gas emissions: Health implications of short-lived greenhouse pollutants. Lancet. 2009;374:2091103. http://dx.doi.org/10.1016/S0140-6736(09)61716-5

15. Solarte I, Caicedo M, Restrepo S. Contaminación atmosférica y enfermedad respiratoria en menores de 14 años en Bogotá. Revista Médica Sanitas. 2002;5:2-21.

16. Secretaría Distrital de Ambiente. Observatorio ambiental de Bogotá. Fecha de consulta: 3 de abril de 2013. Disponible en: http://oab.ambientebogota.gov.co/index. shtml?s=l\&id=532\&v=1.

17. Hernández LJ, Aristizábal G, Quiroz L, Medina K, Rodríguez N, Sarmiento R, et al. Contaminación del aire y enfermedad respiratoria en menores de 5 años de Bogotá. Rev Salud Pública. 2013;15:613-25.

18. Rabinovitch N, Strand M, Gelfand EW. Particulate levels are associated with early asthma worsening in children with persistent disease. Am J Respir Crit Care Med. 2006;173:1098-105. http://dx.doi.org/10.1164/rccm.200509$13930 \mathrm{C}$

19. Speizer FE. Asthma and persistent wheeze in the Harvard Six Cities Study. Chest. 1990;98:191S-5. http://dx.doi. org/10.1378/chest.98.5_Supplement.191S

20. Fernández $\mathbf{M}$, Benítez-Fernández $\mathbf{M}$, Miranda $\mathbf{M}$, Grima F. Validation of the Spanish version of the Phase III ISAAC questionnaire on asthma. J Investig Allergol Clin Immunol. 2005;15:201-10.

21. van Roosbroeck S, Wichmann J, Janssen NA, Hoek G, van Wijnen $\mathrm{JH}$, Lebret $\mathrm{E}$, et al. Long-term personal exposure to traffic-related air pollution among school children, a validation study. Sci Total Environ. 2006;368: 565-73. http://dx.doi.org/10.1016/j.scitotenv.2006.03.034

22. Clark N, Demers P, Karr C, Koehoorn M, Lencar C, Tamburic L, et al. Effect of early life exposure to air pollution 
on development of childhood asthma. Environ Health Perspect. 2010;118:284-90. http://dx.doi.org/10.1289/ehp. 0900916

23. McCormack MC, Breysse PN, Matsui EC, Hansel NN, Williams D, Curtin-Brosnan J, et al. In-home particulate matter concentrations and childhood asthma morbidity. Environ Health Perspect. 2009;117:294-8. http://dx.doi. org/10.1289/ehp. 11770

24. Pérez S, Alfonso J, Amat A, Chofre A, Lucas E, Bou R, et al. Incidencia y factores de riesgo de bronquitis sibilantes en los primeros 6 meses de vida en una cohorte de Alzira (Valencia). An Pediatr (Barc). 2009;72:19-29 http://dx.doi. org/10.1016/j.anpedi.2009.09.007

25. Koehoorn M, Karr C, Demers P, Lencar C, Tamburic L, Brauer M. Descriptive epidemiological features of bronchiolitis in a population-based cohort. Pediatrics. 2008;122: 1196-203. http://dx.doi.org/10.1542/peds.2007-2231

26. Öberg M, Jaakkola M, Woodward A, Peruga A, PrüssUstün A. Worldwide burden of disease from exposure to second-hand smoke: A retrospective analysis of data from 192 countries. Lancet. 2011;377:139-46. http://dx.doi. org/0.1016/S0140-6736(10)61388-8

27. Herrera AB, Rodríguez LA, Niederbacher J. Contaminación biológica intradomiciliaria y su relación con síntomas respiratorios indicativos de asma bronquial en pre-escolares de Bucaramanga, Colombia. Biomédica. 2011;31:357-71. http://dx.doi.org/10.7705/biomedica.v31i3.364

28. Villamizar LA, Herrera AB, Castro $H$, Niederbacher J, Vera LM. Incidencia de síntomas respiratorios y su asociación con contaminación atmosférica en preescolares: un análisis multinivel. Cad Saúde Pública. 2010;26:1411-8. http://dx.doi.org/10.1590/S0102-311X2010000700020

29. Acevedo C, Latorre F, Cifuentes L, Díaz-Martínez L, Garza O. Influencia de la lactancia materna y la alimentación en el desarrollo de alergias en niños. Aten Primaria. 2009;41:67580. http://dx.doi.org/10.1016/j.aprim.2009.04.005

30. Programa de Naciones Unidas para el Desarrollo (PNUD). Informe de Desarrollo Humano 2008, Bogotá, una apuesta por Colombia. Fecha de consulta: 25 de marzo de 2015. Disponible en: http://hdr.undp.org/sites/default/files/ idh_2008_bogota.pdf.

31. Hambling T, Weinstein P, Slaney D. A review of frameworks for developing environmental health indicators for climate change and health. Int J Environ Res Public Health. 2011;8:2854-75. http://dx.doi.org/10.3390/ijerph80x000x

32. Alexeef G, Faust J, August LM, Milanes C, Randles K, Zeise L, et al. A screening method for assessing cumulative impacts. Int J Environ Res Public Health. 2012;9:648-59. http://dx.doi.org/10.3390//ijerph902064 